\title{
Inmunoexpresión de E-cadherina y Vimentina en Mucosa Oral Normal, Displasia Epitelial Oral y Carcinoma Oral de Células Escamosas
}

\author{
Immunoexpression of E-cadherin and Vimentin in Normal Oral Mucosa, \\ Oral Epithelial Displasia and Oral Squamous Cell Carcinoma
}

\author{
Natalia Santibáñez ${ }^{1}$; Alejandra Fernández ${ }^{1,2}$; Javier Fernández ${ }^{3}$; \\ René Martínez ${ }^{1}$; Juan Pablo Fawaz ${ }^{1}$; Sergio Olate ${ }^{4}$ \& Ziyad S. Haidar ${ }^{2,5,6,7}$
}

SANTibÁñez, N.; FernándeZ, A.; FERnÁndeZ, J.; MARTíneZ, R.; FAWAZ, J. P.; OLATE, S. \& HAIDAR Z. Inmunoexpresión de E-cadherina y Vimentina en mucosa oral normal, displasia epitelial oral y carcinoma oral de células escamosas. Int. J. Morphol., 35(2):596-602, 2017.

RESUMEN: El objetivo fue evaluar la inmunoexpresión de E-cadherina y Vimentina en mucosa oral normal (MON), displasia epitelial oral (DEO) y carcinoma oral de células escamosas (COCE). Se realizó un estudio descriptivo de una serie de casos analizandolos mediante técnica de inmunohistoquímica contra E-cadherina y Vimentina 16 muestras de MON, 16 de DEO y 19 de COCE. La inmunotinción fue evaluada cualitativamente considerando extensión e intensidad para E-cadherina e intensidad para Vimentina. El análisis de la extensión e intensidad de la inmunotinción de E-cadherina y Vimentina según diagnóstico reveló una asociación estadísticamente significativa ( $<<0,001)$. Siendo la expresión de E-cadherina más alta en MON, seguido por DEO y más baja en COCE, inversamente a lo que se observó con Vimentina. El presente estudio reveló la subregulación del marcador molecular E-cadherina junto con la expresión aberrante por parte de células epiteliales del marcador mesenquimal Vimentina en muestras de MON, DEO y COCE.

PALABRAS CLAVE: Neoplasias orales; Transición epitelio-mesénquima; Cadherinas; Vimentina; Inmunohistoquímica.

\section{INTRODUCCIÓN}

Dentro del grupo de cánceres que con mayor frecuencia afecta a la cavidad oral, se encuentra el carcinoma oral de células escamosas (COCE), correspondiendo al $90 \%$ de las neoplasias orales (Weatherspoon et al., 2015). El COCE es una neoplasia epitelial invasiva que presenta distintos grados de diferenciación y a pesar de encontrarse en un sitio anatómico de fácil acceso para la inspección visual, su diagnóstico se realiza en etapas avanzadas de su desarrollo. Presenta una tendencia a desarrollar metástasis linfáticas tempranamente siendo éstas, responsable de 145.000 muertes al año (Monteiro de Oliveira Novaes \& William Jr., 2016). Un COCE puede surgir a partir de la transformación maligna de la mucosa oral normal (MON) o bien de una lesión potencialmente maligna (LPM) que a su vez presenta distintos grados de displasia epitelial (DEO) (Ries et al., 2013).

El mecanismo que permite la invasión de las células epiteliales neoplásicas al tejido conectivo subyacente y posteriormente a otros órganos, se ha asociado a la transición epitelio mesénquima (TEM). La TEM es un proceso por medio del cual una célula epitelial sufre cambios bioquímicos para asumir un fenotipo mesenquimal (Lamouille et al., 2014). La TEM se asocia a procesos fisiológicos como la embriogénesis y reparación, así como al COCE (Kotiyal \& Bhattacharya, 2016). En este último la célula epitelial neoplásica modifica sus componentes de adhesión celular y

\footnotetext{
${ }^{1}$ Facultad de Odontología, Universidad Andrés Bello, Chile.

${ }^{2}$ Biomaterials \& Tissue Engineering Research Group (BioMAT'X), Centro de Investigación Biomédica, Universidad de los Andes, Chile.

${ }^{3}$ Facultad de Medicina, Universidad de Chile, Santiago, Chile.

${ }^{4}$ División de Cirugía Oral y Maxilofacial, Universidad de La Frontera, Temuco, Chile.

${ }^{6}$ Facultad de Medicina, Universidad de los Andes, Chile.

${ }^{6}$ Facultad de Odontología, Universidad de los Andes, Chile.

${ }^{7}$ Programa de Mejoramiento Institucional (PMI), I+D+i, Dirección de Innovación, Universidad de los Andes, Chile.
} 
adquiere características de célula mesenquimal, lo que le confiere una mayor capacidad para migrar e invadir sitios anatómicos lejanos al sitio de origen (Benedetti \& Reyes, 2015).

Los marcadores epiteliales identificados durante la TEM asociada a progresión tumoral han sido estudiados en carcinoma de mama, pulmón, colorrectal y cervical (Heerboth et al., 2015) mediante técnicas de inmunohistoquímica. Dentro de éstos, destaca E-cadherina y Vimentina, moléculas considerada un prototipo del proceso (Natarajan et al., 2014). E-cadherina es una proteína transmembrana que corresponde a un miembro del subgrupo de cadherinas clásicas o tipo I y forma parte de las estructuras adhesivas intercelulares llamadas uniones adherentes (Ivanov \& Naydenov, 2013). Por su parte, Vimentina corresponde a una proteína ampliamente expresada y conservada del tercer grupo de filamentos intermedios del citoesqueleto. En adultos, es expresada en tejido conectivo mesenquimal, SNC, músculo, células precursoras pancreáticas y neuronales, entre otras (Satelli \& Li, 2011).

En TEM asociada a cáncer la expresión de Ecadherina y Vimentina es modificada por mecanismos como la sobreexpresión de factores represores de la transcripción, mutaciones en la línea germinal, silenciamiento epigenético y modificaciones postraduccionales (Serrano-Gomez et al., 2016). Lo que genera una subexpresión del marcador epitelial E-cadherina en células neoplásicas y un aumento del marcador mesenquimal Vimentina en las mismas.

En cavidad oral, ha sido estudiada la expresión de Ecadherina y Vimentina en muestras de COCE, sin embargo Akthar agregó la observación de muestras de LPM, abarcando muestras de histología normal y displasia (Balasundaram et al., 2014; Costa et al., 2015; Zhou et al., 2015; Akhtar et al., 2016). Por el contrario, nuestro estudio consideró en el grupo de LPM sólo muestras con diagnóstico de DEO en sus distintos grados. Por lo tanto, el objetivo del presente estudio es evaluar la inmunoexpresión de Ecadherina y Vimentina en mucosa oral normal (MON), displasia epitelial oral (DEO) y carcinoma oral de células escamosas (COCE).

\section{MATERIAL Y MÉTODO}

Se realizó un estudio descriptivo de serie de casos el que contó con la aprobación del Comité de Bioética de la Universidad Andrés Bello (Folio N001), año 2017. Todas las muestras fueron obtenidas de los registros del servicio de anatomía patológica de la facultad de odontología de la
Universidad Andrés Bello, Viña del Mar, entre los años 2004 y 2012. Se contó con la aprobación y firma del director y tratante del servicio.

Se obtuvieron 16 muestras de MON diagnosticados histológicamente adyacente a fibromas irritativos, verrugas vulgares, mucoceles, tatuajes de amalgama y nevos; 16 muestras de DEO (11 displasia leve; 3 displasia moderada y 2 displasia severa) y 19 de COCE ( 8 bien diferenciados; 11 moderadamente diferenciados) que contaron con la información relativa a edad y género del paciente, además de la localización de la lesión. Se descartaron las laminillas histológicas de MON, DEO y COCE que presentaron artefactos de técnica histológica o con ausencia de tejido conjuntivo en la zona adyacente a la displasia epitelial o COCE. Las variables a considerar fueron edad, género, localización, diagnóstico, extensión e intensidad de la inmunotinción.

A partir de 51 muestras previamente incluidas en parafina, se obtuvieron cortes de 4 micrones de espesor que fueron montados en portaobjetos xilanizados, desparafinados y rehidratados por inmersión en diluciones de etanol en series graduadas de forma decreciente. Se realizó un proceso de recuperación antigénica durante $80 \mathrm{mi}$ nutos con CC1 (acondicionador Cell Conditioning Solution1) a pH 8 para E-cadherina y durante 30 minutos para Vimentina. Los cortes de tejido fueron incubados con el anticuerpo monoclonal anti-E-cadherina (DAKO) durante 38 minutos a $37^{\circ} \mathrm{C}$ en una dilución 1:50 y anti-vimentina (Ventana) durante 4 minutos a $37^{\circ} \mathrm{C}$ en una dilución con RTU. El sistema de detección utilizado para E-cadherina fue el kit Optiview Ventana $\left({ }^{\oplus} 2015\right.$ Ventana medical Systems, Inc) y para Vimentina fue el kit Ultraview Ventana $\left({ }^{\odot} 2015\right.$ Ventana medical Systems, Inc), todo el protocolo se realizó utilizando el equipo automatizado BenchMarck GX ( ${ }^{\circ} 2014$ Productos Roche). Finalmente, los cortes fueron deshidratados y montados con cubreobjetos usando entellán como medio de montaje.

El análisis de las muestras fue realizado por dos patólogos orales, doble ciego, previamente estandarizados y calibrados. Se consideraron células epiteliales inmunopositivas para E-cadherina aquellas que presentaron un patrón de tinción de membrana plasmática e inmunopositivas para Vimentina aquellas que presentaron un patrón de tinción citoplasmático, ambos determinados mediante la comparación con controles positivos y negativos insertos en cada una de las láminas. La determinación de la intensidad de inmunotinción para ambos marcadores en MON, DEO y COCE fue evaluada cualitativamente de manera ordinal, en 0: ausencia de tinción; 1+: tinción leve; 2+: tinción moderada; 3+: tinción marcada. Por otra parte, 
la determinación de la extensión (Haidar et al., 2010; Akhtar et al.) de la inmunotinción para E-cadherina en el espesor epitelial de MON, DEO y COCE fue evaluada cualitativamente y categorizada en $1=1-25 \% ; 2=26-50$ $\% ; 3=51-75 \% ; 4=>75 \%$.

La variable edad se representó mediante mediana y rango intercuartílico. Las variables cualitativas (sexo, localización, extensión e intensidad) se representaron mediante frecuencia absoluta y relativa. Para el análisis de la extensión y/o intensidad según diagnóstico se utilizó el test de Kruskal-Wallis y para evaluar las diferencias en el promedio de rangos, se utilizó el test post hoc de Conover -Iman. Se utilizó un nivel de significancia de un 0,05 y se realizó el análisis estadístico con el programa STATA $12^{\circledR}$ (StataCorpLP, Texas, USA).

\section{RESULTADOS}

Los datos relativos al número de muestras incluidas en el estudio, edad y género para cada diagnóstico y en la totalidad de la muestra, se observa en la Tabla I. La exten- sión e intensidad de la expresión de E-cadherina según diagnóstico se observa en la Figura 1 y Tabla II. El análisis de la extensión de E-cadherina según diagnóstico indica que hay diferencias al comparar los 3 grupos en estudio ( $p<0,0022)$. La totalidad de las muestras de MON presentaron extensión 4, mientras que el $81,25 \%$ de las muestras de DEO y un $47,37 \%$ de COCE presentaron la misma extensión. Los resultados obtenidos difieren entre MON y COCE $(p=0,002)$ y entre DEO y COCE $(p=0,0107)$. Sin embargo, no hubo diferencias entre MON y DEO ( $\mathrm{p}=0,07)$. El análisis de la intensidad de E-cadherina según diagnóstico indica que hay diferencias entre los 3 grupos ( $p<0,0001$ ). La totalidad de las muestras de MON presentaron intensidad 3, mientras que un $37,50 \%$ de las muestras de DEO y un $21,05 \%$ de COCE presentaron la misma intensidad. Las resultados de MON difieren de DEO $(p<0,001)$ y de COCE $(p<0,001)$. Sin embargo, no hubo diferencias entre DEO y COCE $(\mathrm{p}=0,058)$.

Al correlacionar la extensión e intensidad de Ecadherina se obtuvo una asociación significativa positiva con un rho de Spearman de 0,5413. Lo que indica que al aumentar la extensión del marcador, también aumenta su intensidad.
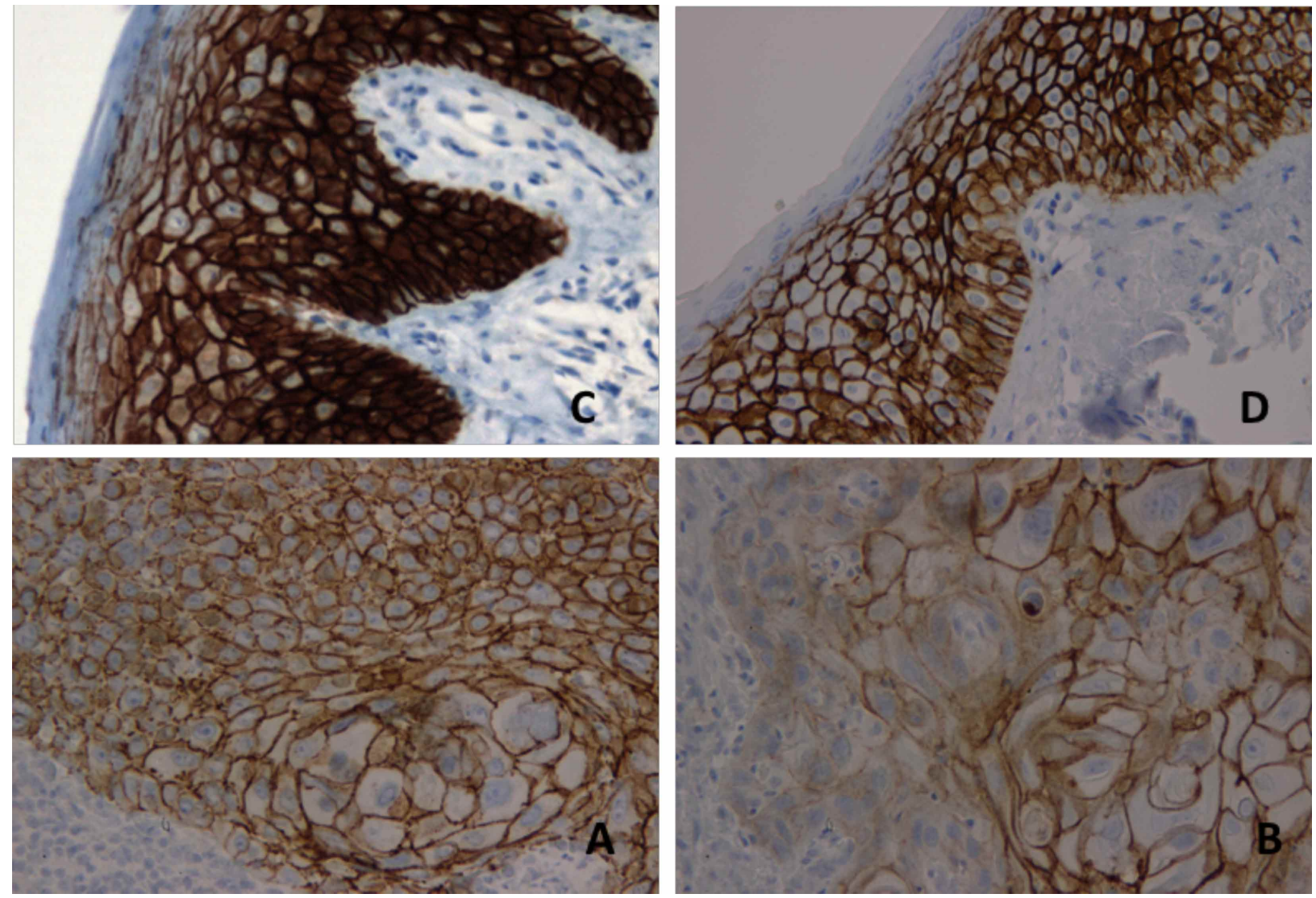

Fig. 1. Expresión de extensión e intensidad de E-cadherina (café) en mucosa oral normal (A); displasia epitelial oral (B), carcinoma oral de células escamosas bien diferenciado (C) y moderadamente diferenciado (D). 40x. 
La intensidad en la expresión de Vimentina según grupo de estudio se muestra en la Figura 2 y Tabla III. Se encontró una asociación estadísticamente significativa entre la intensidad y el diagnóstico (p<0,0002). El $81,25 \%$ de las muestras de MON fueron negativas para el marcador, mientras que un $37,50 \%$ de las muestras de DEO presentaron intensidad 2 y un 36,84\% de las muestras de COCE presentaron intensidad 3. Los resultados obtenidos difieren entre MON y DEO ( $<<0,0203)$, entre MON y COCE $(p<0,000)$ y entre DEO y COCE $(p<0,0044)$.

Existe una asociación significativa negativa con un rho de Spearman de -0,4849 al correlacionar la intensidad de E-cadherina con la de Vimentina, lo que indica que al aumentar la intensidad de la inmunotinción de uno de los marcadores, disminuye la del otro.

Tabla I. Distribución de la muestra por características clínicas y demográficas.

\begin{tabular}{lccccc}
\hline & $\begin{array}{l}\text { Número de } \\
\text { casos }\end{array}$ & $\begin{array}{l}\text { Edad promedio } \\
\text { (años) }\end{array}$ & Rango etario & $\begin{array}{l}\text { Frecuencia } \\
\text { Mujeres \% }\end{array}$ & $\begin{array}{c}\text { Frecuencia } \\
\text { Hombres \% }\end{array}$ \\
\hline MON & 16 & 22,25 & $14-36$ & 68,75 & 31,25 \\
DEO (n:16) & 11 & 55,12 & $37-86$ & 50 & 50 \\
Leve & 3 & & & & \\
Moderada & 2 & & & & 42,11 \\
Severa & 8 & 72,78 & $47-94$ & 57,89 & \\
COCE (n:19) & 11 & & & & \\
Bien diferenciado & 0 & & & & \\
Mod. diferenciado & 51 & 51,39 & $14-94$ & 58,82 & 41,18 \\
Mal diferenciado & & & & & \\
Totalidad de la muestra & & & & & \\
\hline
\end{tabular}

MON: Mucosa oral normal; DEO: Displasia epitelial oral; COCE: Carcinoma oral de células escamosas.
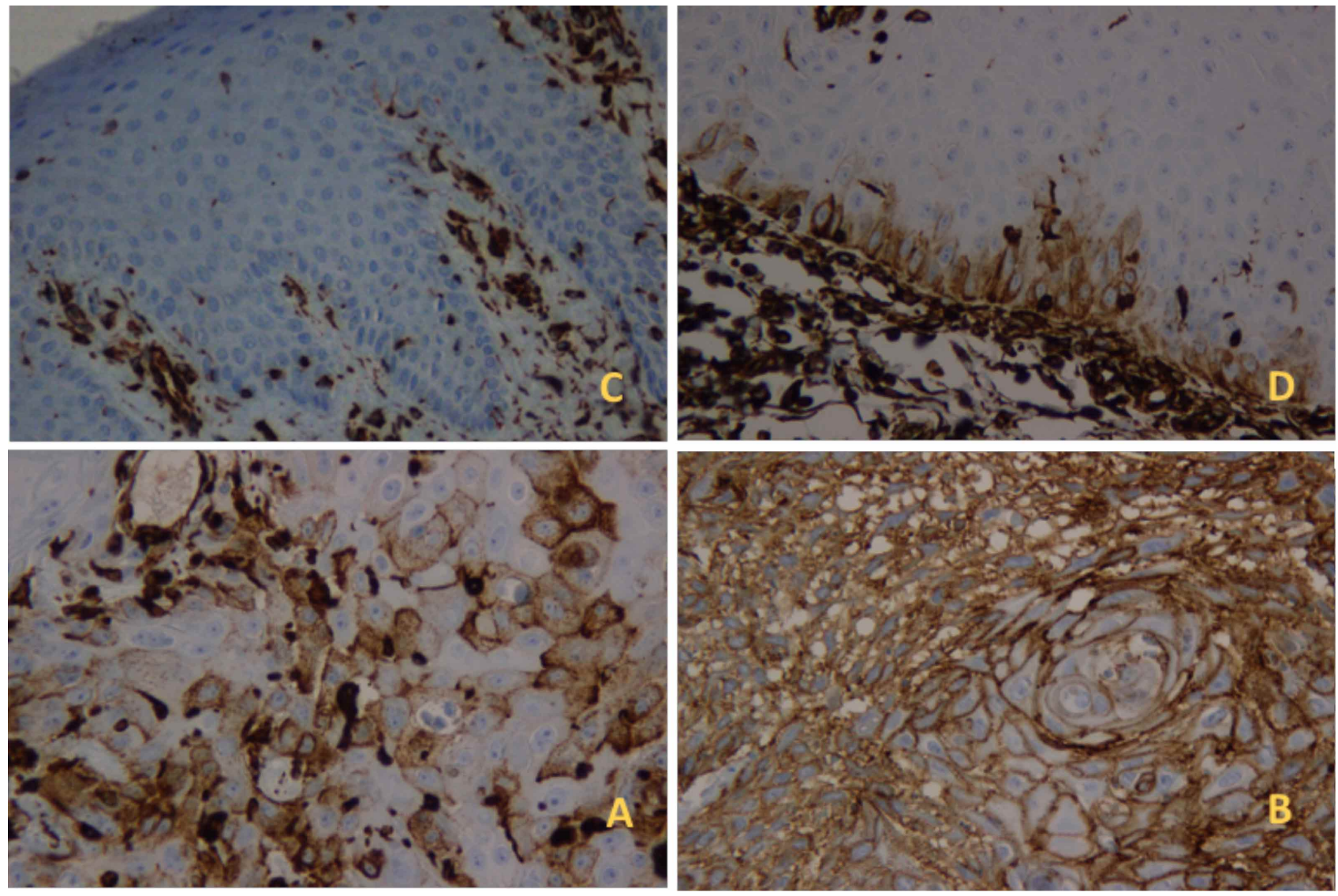

Fig. 2. Expresión de intensidad de Vimentina en mucosa oral normal (A), displasia epitelial oral (B), carcinoma oral de células escamosas moderadamente diferenciado (C) y carcinoma oral de células escamosas bien diferenciado (D). 40x. 
SANTIBÁÑEZ, N.; FERNÁNDEZ, A.; FERNÁNDEZ, J.; MARTíNEZ, R.; FAWAZ, J.P.; OLATE, S. \& HAIDAR Z. Inmunoexpresión de E-cadherina y Vimentina en mucosa oral normal, displasia epitelial oral y carcinoma oral de células escamosas. Int. J. Morphol., 35(2):596-602, 2017.

Tabla II. Distribución de extensión e intensidad de E-cadherina según diagnóstico.

\begin{tabular}{|c|c|c|c|c|c|c|c|c|c|c|c|c|}
\hline & \multicolumn{6}{|c|}{ Extensión } & \multicolumn{6}{|c|}{ Intensidad } \\
\hline & \multirow{2}{*}{\multicolumn{2}{|c|}{$\begin{array}{c}2 \\
26-50 \%\end{array}$}} & \multirow{2}{*}{\multicolumn{2}{|c|}{$\begin{array}{c}3 \\
51-75 \%\end{array}$}} & \multirow{2}{*}{\multicolumn{2}{|c|}{$\begin{array}{c}4 \\
76-100 \%\end{array}$}} & \multirow{2}{*}{\multicolumn{2}{|c|}{$\begin{array}{c}1 \\
\text { leve }\end{array}$}} & \multirow{2}{*}{\multicolumn{2}{|c|}{$\begin{array}{c}2 \\
\text { moderada }\end{array}$}} & \multirow{2}{*}{\multicolumn{2}{|c|}{$\begin{array}{c}3 \\
\text { intensa }\end{array}$}} \\
\hline & & & & & & & & & & & & \\
\hline & $\mathrm{n}$ & $(\%)$ & $\mathrm{n}$ & $(\%)$ & $\mathrm{n}$ & $(\%)$ & $\mathrm{n}$ & $(\%)$ & $\mathrm{n}$ & $(\%)$ & $\mathrm{n}$ & $(\%)$ \\
\hline MON & - & - & - & - & 16 & $(100)$ & - & - & - & - & 16 & $(100)$ \\
\hline DEO & 1 & $(6,25)$ & 2 & $(12,5)$ & 13 & $(81,25)$ & & & 10 & $(62,5)$ & 6 & $(37,50$ \\
\hline COCE & - & - & 10 & $(52,63)$ & 9 & $(47,37)$ & 2 & $(10,53)$ & 13 & $(68,42)$ & 4 & $(21,05$ \\
\hline
\end{tabular}

MON: Mucosa oral normal; DEO: Displasia epitelial oral; COCE: Carcinoma oral de células escamosas.

Tabla III. Distribución de la intensidad de Vimentina según diagnóstico.

\begin{tabular}{lllccccccc}
\multicolumn{10}{c}{ Intensidad } \\
\hline & $\mathrm{n}$ & \multicolumn{1}{c}{$\begin{array}{c}\text { (\%) } \\
\end{array}$} & $\mathrm{n}$ & $(\%)$ & $\mathrm{n}$ & $(\%)$ & $\mathrm{n}$ & $(\%)$ \\
MON & 13 & $(81,25)$ & - & - & 3 & $(18,75)$ & - & - \\
DEO & 6 & $(37,50)$ & 4 & $(25)$ & 6 & $(37,50)$ & - & - \\
COCE & 2 & $(10,53)$ & 5 & $(26,32)$ & 5 & $(26,32)$ & 7 & $(36,84)$ \\
\hline
\end{tabular}

MON: Mucosa oral normal; DEO: Displasia epitelial oral; COCE: Carcinoma oral de células escamosas.

\section{DISCUSIÓN}

La muestra de trabajo reveló que el género no está relacionado con el diagnóstico de $\mathrm{DEO}$ y $\operatorname{COCE}(\mathrm{p}=0,557)$, difiriendo con resultados obtenidos por Martínez et al. (2016) quien reporta una mayor frecuencia de DEO en el género femenino en muestras obtenidas de mucosa oral y faríngea, argumentando que mujeres consultan con mayor anticipación que hombres. Al no encontrar diferencias en cuanto al género más afectado en el grupo de COCE, discrepamos con lo propuesto por la literatura, en donde se indica que el género masculino se ve más afectado, sugiriendo que en la mayoría de los países los hombres están más expuestos que las mujeres a estilos de vida poco saludables y a factores carcinogénicos como alcohol, tabaco y dieta los cuales han sido asociados a la patogénesis de la enfermedad (Weatherspoon et al.; Dholam \& Chouksey, 2016). Sin embargo, nuestros resultados podrían fundamentarse en que en Chile las mujeres se han expuesto crecientemente en la última década a dichos factores, disminuyendo la brecha entre ambos géneros.

Con respecto al rango etario, existe similitud entre nuestros hallazgos y los reportados en la literatura, siendo claro que las DEO anteceden a la edad de diagnóstico del COCE (Dost et al., 2014; Fitzpatrick et al., 2014), probablemente este hecho se deba a que ambas patologías están asociadas a los mismos factores carcinogénicos, siendo fundamental en el COCE el paso del tiempo.

El análisis cualitativo para E-cadherina reflejó que la totalidad de la muestra compuesta por MON presentó una extensión en todo su espesor del inmunomarcador, con un patrón de tinción de membrana citoplasmática que iba decreciendo a medida que las capas celulares se hacían más superficiales. Un 81,25\% de las muestras de DEO y sólo un 47,37 \% de las muestras de COCE obtuvieron la misma extensión. Con respecto a la intensidad de la expresión de Ecadherina, la totalidad de las muestras de MON, presentaron intensidad máxima o marcada, no así las muestras constituidas por DEO y COCE, en que un $62,5 \%$ y un $68,42 \%$ de las muestras respectivamente, presentaron intensidad moderada o media. A partir del análisis de que a medida que progresa el grado de malignidad de la lesión, es decir, desde MON, pasando por DEO hasta COCE, disminuye la extensión e intensidad de la expresión de E-cadherina, podemos inferir que las células están sufriendo TEM, proceso fundamental durante la oncogénesis en donde existe una disminución en la expresión de las uniones epiteliales y la tendencia a adquirir un fenotipo mesenquimal que les conferirá una mayor capacidad para migrar a sitios distantes. Hallazgos similares registran Balasundaram et al., Afrem (2014) y Pereira et al. (2016) quienes reportan una expresión disminuida de E-cadherina en células tumorales en comparación con la fuerte expresión de la misma molécula en MON.

Diversos autores suelen asociar la expresión de Ecadherina por parte de las células tumorales, al grado de diferenciación de la lesión, es así como Zhou et al. sugieren que la baja expresión de E-cadherina en COCE se asocia a un tipo histológico indiferenciado y a una alta frecuencia de generar metástasis linfáticas. Por su parte, Costa et al. pro- 
ponen que tumores con una morfología epitelial conservada es decir, bien diferenciados, presentarían una alta expresión de E-cadherina. Incluso Ren et al. (2016) proponen que la alta expresión de E-cadherina se asocia con buen pronóstico y una mejor sobrevida para carcinomas de cabeza y cuello; tales hallazgos no fueron posibles de observar en nuestro estudio, debido al limitado número de muestras y a no contar con especímenes correspondientes a COCE mal diferenciado, sumado al desconocimiento de la variable presencia o ausencia de metástasis.

Es importante mencionar que la forma en que el estudio fue realizado podría influir en los resultados obtenidos, lo que explicaría algunas discrepancias con publicaciones anteriores, ya que algunos de ellos utilizaron una estimación semi-cuantitativa al momento de realizar el análisis de los marcadores. Incluso el área analizada del tumor difiere en los distintos estudios, siendo en algunos de ellos evaluado el frente de invasión del tumor y en otros, áreas al azar.

El análisis cualitativo para Vimentina reflejó en el grupo de MON intensidad 0 en la mayoría de los casos $(81,25$ $\%)$. Estas observaciones son coincidentes con lo reportado por Zhou et al. Adicionalmente, se observó la expresión positiva de Vimentina en células del tejido conectivo submucoso y en el citoplasma de células mesenquimales del estroma, característica considerada normal para este marcador. Sin embargo, el 18,75 \% de las muestras de MON presentaron una intensidad moderada de Vimentina, lo que nos permite inferir que el componente epitelial de la mucosa oral estaría sufriendo cambios moleculares en ausencia de alteraciones fenotípicas compatibles con displasia epitelial, sugiriendo que la transformación maligna puede surgir a partir de MON, lo que coincide con lo descrito en la literatura (Fleskens \& Slootweg, 2009).

A diferencia del grupo de MON, la inmunoexpresión de Vimentina en el 37,5 \% de las muestras del grupo DEO presentó una intensidad moderada, lo que puede deberse a una disminución en la expresión de uniones epiteliales generando la pérdida parcial o total de polaridad celular, junto con un aumento en la expresión del marcador mesenquimal dando inicio a la migración celular en un patrón irregular sin traspasar aún la membrana basal.

En las muestras de COCE, encontramos que la intensidad de Vimentina en la mayoría de los casos fue marcada $(36,84 \%)$. Lo mismo ocurre en carcinomas prostáticos, gástricos, colorrectales, mamarios, cérvico-uterino y COCE en hallazgos reportados por Satelli \& Li, Costa et al. y Whiteland et al. (2013) entre otros, quienes asocian esta sobre expresión con tumores pobremente diferenciados y con un fenotipo invasivo. Esta sobre expresión responde a la regulación que sufre el gen que codifica Vimentina, ya sea por medio de activadores transcripcionales, modificaciones epigenéticas o modificaciones postraduccionales. De aquí se podría inferir que la intensidad pesquisada para Vimentina en los carcinomas, a diferencia de los grupos MON y DEO, estaría revelando un proceso de reprogramación celular por medio del cual, las células epiteliales neoplásicas adquieren un fenotipo mesenquimal, forzándolas a cambiar su morfología y adquirir movilidad, permitiéndoles traspasar la membrana basal, invadir el tejido conectivo subepitelial e ingresar a vasos linfáticos o sanguíneos.

Debemos considerar algunas limitaciones en el presente estudio, la principal de ellas fue el número de muestras obtenidas, en comparación con estudios realizados anteriormente por Balasundaram et al. que incluyen aproximadamente 60 casos de COCE y Akhtar et al. con 64 muestras de lesiones displásicas. Esto podría deberse a que tanto las displasias y carcinomas orales de baja frecuencia. Sumado a lo anterior, no contamos con muestras de COCE mal diferenciado, ni datos del estadio clínico del paciente o seguimiento posterior, los que serían útiles para que en estudios futuros sea posible relacionar la TEM con el pronóstico de los pacientes.

\section{CONCLUSIÓN}

El presente estudio reveló la subregulación del marcador molecular E-cadherina en células epiteliales en muestras de DEO y COCE, junto con la expresión aberrante de Vimentina en células epiteliales de muestras de MON, DEO y COCE.

SANTIBÁÑEZ, N.; FERNÁNDEZ, A.; FERNÁNDEZ, J.; MARTÍNEZ, R.; FAWAZ, J. P.; OLATE, S. \& HAIDAR Z. Immunoexpression of E-cadherin and Vimentin in normal oral mucosa, oral epithelial displasia and oral squamous cell carcinoma. Int. J. Morphol., 35(2):596-602, 2017.

SUMMARY: The aim was to evaluate the expression of E-cadherin and Vimentin in oral epithelial dysplasia (OED) and oral squamous cell carcinoma (OSCC), in comparison with normal oral mucosa (NOM) in a descriptive case study using immunohistochemistry. A total of fifty-one $(\mathrm{N}=51)$ histological samples were included; as follows: $\mathrm{n}=16(\mathrm{NOM}), \mathrm{n}=16(\mathrm{OED})$ and $\mathrm{n}=19$ (OSCC). All samples were analyzed using immunohistochemistry against the expression of E-cadherin and Vimentin. Immunostaining was qualitatively evaluated by extent and intensity of its expression for E-cadherin and intensity for Vimentin. Extension and intensity analysis of E-cadherin and 
Vimentin immunostaining according to group revealed a statistically significant association $(\mathrm{r}<0.001)$. E-cadherin expression was found to be highest in NOM followed by OED and lowest in OSCC, inverse to what was observed with Vimentin. The present study revealed the down regulation of the molecular marker Ecadherin, suggestive of reduction in dysplastic cells on comparison to NOM cells, and aberrant expression of the mesenchymal marker Vimentin by epithelial cells in samples of NOM, OED and OSCC; questioning their value as a prognostic marker.

\section{KEY WORDS: Oral neoplasms; Epithelial mesenchymal transition; Cadherins; Vimentin; Immunohistochemistry.}

\section{REFERENCIAS BIBLIOGRÁFICAS}

Afrem, M. C.; Margaritescu, C.; Crait „oiu, M. M.; Ciuca, M.; Sarla, C. G. \& Cotoi, O. S. The immunohistochemical investigations of cadherin "switch" during epithelial-mesenchymal transition of tongue squamous cell carcinoma. Rom. J. Morphol. Embryol., 55(3):1049-56, 2014.

Akhtar, K.; Ara, A.; Siddiqui, S. A. \& Sherwani, R. K. Transition of immunohistochemical expression of e-cadherin and vimentin from premalignant to malignant lesions of oral cavity and oropharynx. Oman Med. J., 31(3):165-9, 2016.

Balasundaram, P.; Singh, M. K.; Dinda, A. K.; Thakar, A. \& Yadav, R. Study of b-catenin, E-cadherin and vimentin in oral squamous cell carcinoma with and without lymph node metastases. Diagn. Pathol., 9:145, 2014.

Benedetti, I. \& Reyes, N. Transición epitelial-mesenquimal en la progresión del adenocarcinoma prostático. Iatreia, 28(4):420-33, 2015.

Costa, L. C.; Leite, C. F.; Cardoso, S. V.; Loyola, A. M.; Faria, P. R.; Souza, P. E. \& Horta, M. C. Expression of epithelial-mesenchymal transition markers at the invasive front of oral squamous cell carcinoma. J. Appl. Oral. Sci., 23(2):169-78, 2015.

Dholam, K. P. \& Chouksey, G. C. Squamous cell carcinoma of the oral cavity and oropharynx in patients aged $18-45$ years: A case-control study to evaluate the risk factors with emphasis on stress, diet, oral hygiene, and family history. Indian J. Cancer, 53(2):244-51, 2016.

Dost, F.; Ford, P. J. \& Farah, C. S. Heightened risk of second primary carcinoma of the head and neck following cervical neoplasia. Head Neck, 36(8):1132-7, 2014

Fitzpatrick, S. G.; Honda, K. S.; Sattar, A. \& Hirsch, S. A. Histologic lichenoid features in oral dysplasia and squamous cell carcinoma. Oral Surg. Oral Med. Oral Pathol. Oral Radiol., 117(4):511-20, 2014.

Fleskens, S. \& Slootweg, P. Grading systems in head and neck dysplasia: their prognostic value, weaknesses and utility. Head Neck Oncol., 1:11, 2009.

Haidar, Z. S.; Hamdy, R. C. \& Tabrizian, M. Biocompatibility and safety of a hybrid core-shell nanoparticulate OP-1 delivery system intramuscularly administered in rats. Biomaterials, 31(10):2746-54, 2010.

Heerboth, S.; Housman, G.; Leary, M.; Longacre, M.; Byler, S.; Lapinska, K.; Willbanks, A. \& Sarkar, S. EMT and tumor metastasis. Clin. Transl. Med., 4:6, 2015.

Ivanov, A. I. \& Naydenov, N. G. Dynamics and regulation of epithelial adherens junctions: recent discoveries and controversies. Int. Rev. Cell Mol. Biol., 303:27-99, 2013.

Kotiyal, S. \& Bhattacharya, S. Events of molecular changes in epithelialmesenchymal transition. Crit. Rev. Eukaryot. Gene Expr, 26(2):16371, 2016.

Lamouille, S.; Xu, J. \& Derynck, R. Molecular mechanisms of epithelialmesenchymal transition. Nat. Rev. Mol. Cell. Biol., 15(3):178-96, 2014.
Martínez, C.; Hernández, M.; Martínez, B. \& Adorno, D. Frequency of oral squamous cell carcinoma and oral epithelial dysplasia in oral and oropharyngeal mucosa in Chile. Rev. Med. Chile, 144(2):169-74, 2016.

Monteiro de Oliveira Novaes, J. A. \& William Jr., W. N. Prognostic factors, predictive markers and cancer biology: the triad for successful oral cancer chemoprevention. Future Oncol., 12(20):2379-86, 2016.

Natarajan, J.; Chandrashekar, C. \& Radhakrishnan, R. Critical biomarkers of epithelial-mesenchymal transition in the head and neck cancers. $J$. Cancer Res. Ther. 10(3):512-8, 2014.

Pereira, C. H.; Morais, M. O.; Martins, A. F. L.; Soares, M. Q. S.; Alencar, R. C. G.; Batista, A. C.; Leles, C. R. \& Mendonça, E. F. Expression of adhesion proteins (E-cadherin and b-catenin) and cell proliferation (Ki67) at the invasive tumor front in conventional oral squamous cell and basaloid squamous cell carcinomas. Arch. Oral Biol., 61:8-15, 2016.

Ren, X.; Wang, J.; Lin, X. \& Wang, X. E-cadherin expression and prognosis of head and neck squamous cell carcinoma: evidence from 19 published investigations. Onco Targets Ther, 9:2447-53, 2016.

Ries, J.; Vairaktaris, E.; Agaimy, A.; Bechtold, M.; Gorecki, P.; Neukam, F. W. \& Nkenke, E. The relevance of EGFR overexpression for the prediction of the malignant transformation of oral leukoplakia. Oncol. Rep., 30(3):1149-56, 2013.

Satelli, A. \& Li, S. Vimentin as a potential molecular target in cancer therapy Or Vimentin, an overview and its potential as a molecular target for cancer therapy. Cell Mol. Life Sci., 68(18):3033-46, 2011.

Serrano-Gomez, S. J.; Maziveyi, M. \& Alahari, S. K. Regulation of epithelial-mesenchymal transition through epigenetic and posttranslational modifications. Mol. Cancer, 15:18, 2016.

Weatherspoon, D. J.; Chattopadhyay, A.; Boroumand, S. \& Garcia, I. Oral cavity and oropharyngeal cancer incidence trends and disparities in the United States: 2000-2010. Cancer Epidemiol., 39(4):497-504, 2015.

Whiteland, H.; Spencer-Harty, S.; Thomas, D. H.; Davies, C.; Morgan, C.; Kynaston, H.; Bose, P.; Fenn, N.; Lewis, P. D.; Bodger, O.; Jenkins, S. \& Doak, S. H. Putative prognostic epithelial-to-mesenchymal transition biomarkers for aggressive prostate cancer. Exp. Mol. Pathol., 95(2):2206, 2013.

Zhou, J.; Tao, D.; Xu, Q.; Gao, Z. \& Tang, D. Expression of E-cadherin and vimentin in oral squamous cell carcinoma. Int. J. Clin. Exp. Pathol., 8(3):3150-4, 2015.

Dirección para correspondencia:

Ziyad S. Haidar

BioMAT'X

Facultad de Odontología

Universidad de Los Andes

Mons. Álvaro del Portillo 12.455

Las Condes

Santiago

CHILE

E-mail: zhaidar@uandes.cl

Recibido : 12-03-2017

Aceptado: 30-03-2017 